\title{
RAMÓN JOFFRE Gabriel, Los alfareros golondrinos, productores itinerantes en los Andes
}

\section{Alicia Espinosa}

\section{OpenEdition}

Journals

Édition électronique

URL : https://journals.openedition.org/jsa/16225

DOI : 10.4000/jsa. 16225

ISSN : 1957-7842

Éditeur

Société des américanistes

Édition imprimée

Date de publication : 15 décembre 2018

Pagination : 205-210

ISSN : 0037-9174

\section{Référence électronique}

Alicia Espinosa, « RAmón JOFFRE Gabriel, Los alfareros golondrinos, productores itinerantes en los Andes », Journal de la Société des américanistes [En ligne], 104-2 | 2018, mis en ligne le 15 décembre 2018, consulté le 04 septembre 2022. URL : http://journals.openedition.org/jsa/16225 ; DOl : https:// doi.org/10.4000/jsa. 16225

Ce document a été généré automatiquement le 4 septembre 2022

Tous droits réservés 


\title{
RAMÓN JOFFRE Gabriel, Los alfareros golondrinos, productores itinerantes en los Andes
}

\author{
Alicia Espinosa
}

\section{RÉFÉRENCE}

RAMÓN JOFFRE Gabriel, Los alfareros golondrinos, productores itinerantes en los Andes, Instituto Francés de Estudios Andinos/Sequilao Editores, Lima, 2013, 168 p., bibliogr., append., ill. (en noir et blanc), photos (en noir et blanc), cartes, tabl.

1 Gabriel Ramón Joffre, actuellement professeur à l'université Pontificale du Pérou, possède une double formation en histoire et archéologie, au terme de laquelle il a publié Los alfareros golondrinos (2013). L'ouvrage est un extrait de sa thèse soutenue en 2008, dans laquelle il présentait plus amplement les communautés potières encore existantes dans les Andes péruviennes, à partir d'enquêtes ethnographiques et d'analyses de documents datant des périodes coloniale et actuelle. Le livre ici présenté illustre le cas particulier des alfareros golondrinos, autrement dit des potiers itinérants du Pérou. L'objectif pour l'auteur est de rendre compte de la nature de cette itinérance artisanale et de sa portée à la fois méthodologique et théorique dans le cadre des hypothèses relatives aux lieux et aux modes de production de la céramique. L'ouvrage s'articule en cinq grandes parties où sont progressivement présentés le cadre géographique, puis méthodologique, une définition des potiers itinérants andins, et une proposition de typologie de ce phénomène à partir des différents cas observés. Une réflexion finale autour de la mobilité à l'époque précoloniale est amorcée au cours de la discussion, où ses observations sont mises en perspective avec le registre archéologique.

2 Le qualificatif golondrinos (hirondelles) fait référence aux migrants mexicains qui au $\mathrm{xx}^{\mathrm{e}}$ siècle participaient chaque année à la récolte du coton dans le sud des États-Unis, ainsi qu'aux Italiens qui entre le $\mathrm{xIX}^{\mathrm{e}}$ et le $\mathrm{xx}^{\mathrm{e}}$ siècle se rendaient en Argentine pour les 
moissons, avant de retourner dans leur communauté d'origine après plusieurs mois d'absence (Miller 1964 ; Scobie 1964). Ce terme est mobilisé par l'auteur afin de définir un mode particulier de production artisanale au Pérou, où durant une partie de l'année des potiers, seuls ou accompagnés, parcourent de longues distances afin de se rendre dans d'autres communautés rurales et d'y élaborer leurs céramiques (Sabogal 1987, p. 17-30). Cette mobilité est saisonnière, et coïncide avec le calendrier agricole puisqu'elle a lieu après les récoltes durant la saison sèche, ce qui correspond aux mois de mai-juin à novembre-décembre dans la sierra. Les routes qu'empruntent les potiers ne sont pas aléatoires. Ceux-ci se rendent en effet de façon cyclique dans différentes communautés avec lesquelles ils ont tissé des liens depuis plusieurs générations. Les poteries qu'ils produisent sur place sont le plus souvent échangées contre des denrées agricoles abondantes en cette saison, ce qui explique en retour le besoin des populations en récipients de stockage. Les alfareros golondrinos sont une catégorie bien particulière de potiers, car leur mobilité de leur communauté d'origine entrâne un déplacement des lieux de production. Dans ces communautés de destination, les artisans maintiennent leur style technique (interne), contrairement au style décoratif (externe) qui peut se modifier en fonction de la demande. Cette itinérance implique donc une mobilité des lieux de production, et se distingue en conséquence des autres modes de production, comme celui des potiers de Raqch'i dans la région de Cuzco qui fabriquent leurs céramiques directement dans leur unité familiale pour ensuite les vendre ou les échanger sur les marchés (Mohr 1984, p. 162). L'exemple andin est aussi unique, puisque les potiers emportent leurs propres matières premières durant leurs déplacements (matériaux argileux et/ou dégraissants), contrairement aux potiers itinérants de Chypre et de Crète (Christakis 1998; Ionas 2000), ou encore d'Argentine (Cremonte 1984) qui utilisent des argiles locales.

3 Cependant, bien que plusieurs sources écrites rendent compte de ce phénomène durant la période coloniale et actuelle dans les Andes (Sillar 2009), les alfareros golondrinos sont finalement peu pris en considération, en particulier dans le cadre des interprétations sur la production céramique à l'époque précoloniale. À partir de ses enquêtes, Gabriel Ramón tente de saisir les mécanismes sous-jacents à la mobilité entre les zones rurales. Les questions qu'ils soulèvent sont le plus souvent mises en perspective avec des problématiques propres à l'archéologie, en lien avec la reconnaissance des entités culturelles et des liens qu'elles entretenaient les unes avec les autres. L'auteur insiste ainsi sur la nécessité de déconstruire les modèles nomothétiques communément acceptés, et il réfute en cela le statisme souvent attribué aux communautés traditionnelles des Andes. Au contraire, il considère la mobilité comme une composante essentielle de leur système socio-économique. L'auteur nous pousse alors à envisager des scénarios plus dynamiques que la simple circulation de produits finis, en établissant une distinction entre deux types de production : une production et une distribution locale de céramiques par des artisans originaires de la communauté, et une production faite par des artisans se déplaçant depuis leur communauté d'origine pour produire et/ou distribuer leurs poteries dans d'autres localités.

4 L'essai de typologie des modes de production potière itinérante que propose Gabriel Ramón s'articule autour de quatre critères: les routes et les itinéraires empruntés, les étapes de manufacture pratiquées dans la communauté de destination, les modes de 
distribution des produits finis et l'ampleur de la mobilité (individu seul ou déplacement collectif). De la sorte, il distingue six cas avec leurs variables :

1. Les potiers itinérants qui se rendent régulièrement dans une série de communautés, en alternant chaque année leur destination. Les potiers voyagent seuls et vivent chez leurs hôtes. Ils emportent avec eux leurs matières premières et leurs outils, et se chargent de l'ensemble du processus de production des céramiques qu'ils échangent contre des produits agricoles.

2. Les potiers itinérants se déplaçant, seuls ou accompagnés, dans un nombre réduit de communautés avec lesquelles ils entretiennent des liens privilégiés :

- Les potiers qui entretiennent une relation d'interdépendance avec une zone de haute productivité agricole. Ils voyagent avec leurs outils et leurs matières premières, pour produire sur place et échanger leurs poteries sur le marché. Ils vivent en dehors du village pour réduire le coût du voyage.

- Les artisans qui maintiennent une relation sociale directe avec la même communauté de destination, forgée depuis plusieurs générations. Ces derniers se déplacent uniquement avec leur argile et collectent le dégraissant sur place. Ils s'occupent de la production alors que les hôtes se chargent de la distribution des poteries.

3. Relation d'interdépendance entre un lieu attractif et une zone isolée : le cas des peonas, des potières du nord du Pérou contactées par d'autres artisanes qui requièrent leurs services durant une partie de l'année. L'auteur cite le cas des potières de Yacya (peonas) se rendant à Acopalca (département d'Ancash) où la demande en céramique augmente durant les festivités locales. Les potières de ces deux communautés travaillent donc conjointement, chacune avec sa propre tradition technique, même si les peonas n'effectuent ni la préparation des matières premières, ni la décoration, ni la cuisson et ni la distribution des poteries.

4. Les potiers sédentarisés dans une communauté de destination, entraînant une dispersion des lieux de production. Le cas présenté est celui des potiers de Simbilá (Piura), qui se rendaient régulièrement dans d'autres communautés afin de produire sur place. Aujourd'hui, une partie de cette communauté s'est définitivement installée dans certains lieux où ils produisent encore avec leur style technique d'origine.

5. Déplacements cycliques des potiers entre la sierra et la côte.

6. Les olleros de puna, des pasteurs transhumants, mais aussi artisans, vivant au-delà de $2800 \mathrm{~m}$ d'altitude. Ils produisent leurs poteries dans différentes zones de la puna, ou dans des zones plus basses pour les échanger contre du maïs, du blé et de l'orge. Dans ce cas, les lieux de production et d'habitat sont mobiles.

5 Bien que cette typologie soit préliminaire pour l'auteur, elle montre que mobilité et production ne sont pas des concepts indissociables, comme en témoigne le cas finalement peu isolé des potiers itinérants andins. De la diversité des cas exposés, il en ressort les points suivants. L'itinérance se justifie par le besoin en produits agricoles que les artisans ne cultivent pas en quantités suffisantes dans leur propre communauté, ce qui induit des déplacements au moment des récoltes. Ces voyages réguliers tissent des relations d'interdépendance entre les potiers issus d'une zone à faible potentiel agricole et des hôtes qui eux possèdent des terres à plus haut rendement. Il est intéressant de noter que plus la relation entre les deux communautés est forte, plus l'intervention de l'hôte est importante comme l'illustre l'exemple des peonas. Ce cas démontre aussi à quel point le style externe s'adapte à la demande locale, contrairement aux techniques de façonnage qui elles ne changent pas. L'auteur amorce en conséquence un début de réflexion sur le raisonnement archéologique expliquant la présence de poteries semblables dans un même village, mais pourtant fabriquées par deux communautés 
potières aux traditions techniques distinctes. Comment les archéologues auraient-ils en effet envisagé ce lien, et quels critères auraient-ils employés pour définir leur lieu et leur mode de production?

La discussion finale met en perspective les observations actualistes de l'auteur avec le registre archéologique, afin de définir les potentielles traces laissées par les potiers itinérants en contexte archéologique. D'après lui, le matériau à privilégier serait la céramique utilitaire, et non pas celle communément associée à l'élite, car les critères morpho-stylistiques peuvent s'avérer peu opérants en raison de l'adaptation du style décoratif selon la demande locale. De la sorte, malgré l'absence générale de décor sur les céramiques utilitaires, il envisage ce matériau comme un outil analytique pertinent afin d'aborder l'identité des producteurs en contexte archéologique (Ramón et Bell 2013). Loin d'être le résultat d'une production locale et domestique, ces dernières peuvent en effet être produites à des centaines de kilomètres de leur lieu de consommation, voire même par des potiers issus d'autres régions, venus les fabriquer sur place.

7 Afin de tracer la provenance de ces poteries, Gabriel Ramón signale l'importance de l'identification du style technique qu'il considère comme un marqueur d'identités techniques locales, un travail qui nécessiterait d'élaborer une véritable " géographie des techniques» (p. 46). L'auteur ouvre donc des perspectives d'analyses novatrices pour les Andes, qui permettraient d'entrevoir sous un angle nouveau les communautés de filiation technologique, les contacts entre différentes communautés, tout comme la potentielle reconnaissance des potiers itinérants en contexte précolonial. Objets et producteurs seraient donc considérés en mouvement, puisque comme nous l'avons vu, les lieux de production et de distribution ne correspondent pas forcément aux frontières ethniques. On regrettera toutefois le manque de développement d'un cadre méthodologique afin d'identifier ces techniques, qu'il mentionne néanmoins en proposant la création d'un référentiel des traces laissées par les outils à différentes étapes de la chaîne opératoire (p. 41).

8 À travers l'exemple des alfareros golondrinos, Gabriel Ramón pense une façon nouvelle d'intégrer les thèmes de mobilité artisanale dans le cadre des systèmes socioéconomiques andins, passés et présents. L'ouvrage est une source inédite d'informations quant à l'existence de ces potiers itinérants, et de leurs différentes expressions dans les Andes du Pérou. Il s'inscrit ainsi dans une tendance qui vise à analyser le style technique des producteurs, afin de mieux discerner la composition sociale des communautés précoloniales.

\section{BIBLIOGRAPHIE}

\section{CHRISTAKIS Kostis}

1998 "Craft specialization in Minoan Crete. The case for itinerant pithos makers ", in Bogdan Rutkowski (dir.), Aegean Archaeology, vol. 3, Art and Archaeology (Studies and monographs in mediterranean archaeology and civilization, 2 [4]), Warsaw, p. 63-74. 
CREMONTE Beatriz

1984 « Alfareros itinerantes de Los Colorados (Dto. Tafi, Tucumán), Aproximaciones a un estudio de etnografía arqueológica », Runa, 14, p. 247-261.

IONAS Ioannis

2000 Traditional pottery and potters in Cyprus, The disappearance of an ancient craft industry in the 19th and 20th centuries, Ashgate, Aldershot.

MILLER Charlene

1964 « Los golondrinos », Kroeber Anthropological Society Papers, 30, p. 51-71.

MOHR Karen

1984 « Traditional pottery of Raqch'i, Cuzco, Peru. A preliminary study of its production, distribution, and consumption », Ñawpa Pacha, 22-23, p. 161-210.

RAMÓN JOFFRÉ Gabriel

2008 Potters of the northern Peruvian Andes: a palimpsest of technical styles in motion, thesis submitted in partial fulfilment of the requirements for the degree of Doctor of Philosophy, Sainsbury Research Unit, University of East Anglia.

RAMÓNJOFFRÉ Gabriel et Martha BELL

2013 « Re-placing plainware: production and distribution of domestic pottery, and the narration of the pre-colonial past in the Peruvian Andes ", Journal of Anthropological Archaeology, 32, p. 595-613.

SABOGAL WIESSE José

1987 Cerámica yunga, estribación andino piurana, CIPCA, Piura.

SCOBIE James

1964 Revolution on the pampas : a social history of Argentine wheat, 1860-1910, University of Texas Press, Austin.

SILLAR Bill

2009 « La saisonnalité des techniques », Techniques \& Culture, 52-53, p. 90-119.

\section{AUTEURS}

\section{ALICIA ESPINOSA}

Étudiante de doctorat, Archéologie des Amériques (UMR 8096), université Panthéon-Sorbonne 